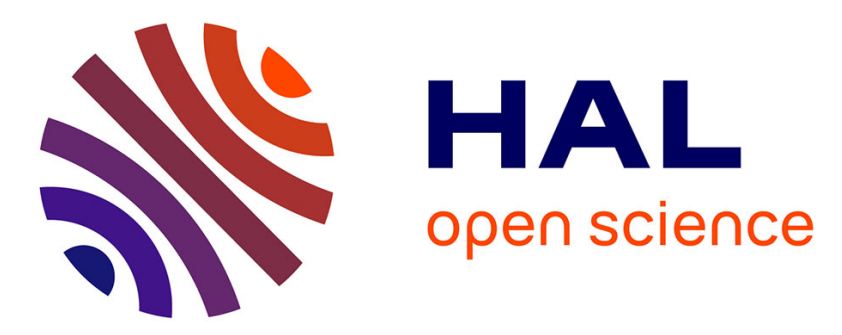

\title{
Monitoring of polymer content in an emulsion polymerization using spatially resolved spectroscopy in the near infrared region and Raman spectroscopy
}

Manis Gheghiani, Noémie Caillol, Serge Henrot, Timothy Mckenna, Nida

Sheibat-Othman

\section{To cite this version:}

Manis Gheghiani, Noémie Caillol, Serge Henrot, Timothy Mckenna, Nida Sheibat-Othman. Monitoring of polymer content in an emulsion polymerization using spatially resolved spectroscopy in the near infrared region and Raman spectroscopy. Polymer Engineering and Science, 2020, 60 (9), pp.2248-2261. 10.1002/pen.25467 . hal-02941351

\section{HAL Id: hal-02941351 \\ https://cnrs.hal.science/hal-02941351}

Submitted on 20 Nov 2020

HAL is a multi-disciplinary open access archive for the deposit and dissemination of scientific research documents, whether they are published or not. The documents may come from teaching and research institutions in France or abroad, or from public or private research centers.
L'archive ouverte pluridisciplinaire HAL, est destinée au dépôt et à la diffusion de documents scientifiques de niveau recherche, publiés ou non, émanant des établissements d'enseignement et de recherche français ou étrangers, des laboratoires publics ou privés. 
Monitoring of polymer content in an emulsion polymerization using spatially resolved spectroscopy in the near infrared region and Raman spectroscopy

Manis Gheghiani ${ }^{1}$, Noémie Caillol ${ }^{2}$, Serge Henrot ${ }^{2}$, Timothy McKenna ${ }^{3}$, Nida Sheibat-Othman ${ }^{1 *}$

${ }^{1}$ University of Lyon, University Claude Bernard Lyon 1, CNRS, LAGEPP UMR 5007, F-69100, Villeurbanne, France

${ }^{2}$ Axel'One, Solaize, France

${ }^{3}$ University of Lyon, Université Claude Bernard Lyon 1, CPE Lyon, CNRS, UMR 5265, C2P2, Villeurbanne, France *Corresponding author: nida.othman@univ-lyon1.fr

\begin{abstract}
The potential of spatially resolved spectroscopy (SRS) for in situ monitoring is evaluated in this work. SRS is based on near-infrared spectroscopy. It is well adapted to heterogeneous systems and collects information about both physical and chemical properties. In this work, the polymer content in emulsion copolymerization is predicted using SRS. The reaction was first carried out in batch mode for particle nucleation followed by semi-continuous monomer addition under starved conditions to allow particle growth. SRS and Raman spectroscopy are compared, and the advantages and disadvantages of both methods are highlighted, revealing that each method has its own benefits. Different operating conditions were varied, including the monomer ratio, the surfactant mass fraction and the agitation speed. Regression models were developed using partial least square for both techniques.
\end{abstract}

\title{
1. Introduction
}

Online monitoring is essential in order to ensure the process security, increase its productivity and control the final product properties. Monitoring can be done at-line by employing a sampling loop, or in-line using in situ probes. The latter method is simpler to install and is more representative of the reaction medium as it does not imply changes in the process characteristics like temperature or shear. Different types of processes in pharmaceutical [1]-[3] and chemical industries [4]-[6] have been successfully monitored online, for both homogeneous and heterogeneous systems.

Emulsion polymerization is a heterogeneous process for which monitoring is essential in order to predict changes in the operating conditions and be able to reconfigure the operation. Usually, it is desired to measure the different concentrations of monomer(s), polymer and surfactant as well as key product properties like the polymer molecular weight or the particle size. However, the multiphase aspect of the reaction medium represents an added challenge for its precise 
monitoring. Calorimetry is historically the most widely used technique for online monitoring of polymerization processes due to the exothermic nature of the reaction. For instance, it has been used to monitor the monomer conversion and the copolymer composition in butyl acrylatestyrene, acetate-butyl acrylate [7] or vinyl acetate-butyl acrylate [8] copolymerization systems, and to monitor the reaction rate in emulsion polymerization of styrene [9]. The conductivity was also introduced to monitor the concentrations of ionic species and their partitioning between the different phases, and thereby to deduce the different reaction steps in emulsion polymerization (i.e. nucleation, growth of particles, coagulation, and monomer-saturated and unsaturated intervals) [10]. However, monitoring by conductivity is limited for ionic surfactant.

Spectroscopy has increasingly been used for online monitoring of polymerization systems, mainly Raman, mid infrared (MIR) and near infrared (NIR) spectroscopies [11], [12]. Such techniques use probes that can be inserted in an existing reactor with no need of sampling. Raman spectroscopy has been widely used in emulsion polymerization due to the low interference of water in the measurement [13], [14]. For instance, Elizalde et al. (2004) showed that Raman spectroscopy provides better results, under starved conditions and high solid content, than calorimetry in terms of monitoring of the monomer conversion and free monomer concentration in the copolymerization of vinyl acetate/butyl acrylate and butyl acrylate/methyl methacrylate [15]. Regarding NIR spectroscopy, it provides information similar to MIR spectroscopy with the advantage of being more energetic and so it is easier to implement online but it generates overlapping and harmonic vibrations which are more complex to interpret and therefore requires the use of advanced chemometrics to treat the data. Gossen \& Al (1993) [16] were among the first to use it to predict the conversion in a copolymerization process of styrene and methyl methacrylate.

NIR spectra contain chemical information like the monomer or polymer concentrations (from which the monomer conversion can be calculated) [17] as well as some physical information related to the size and morphology of particles since it is sensitive to the diffusion of photons in the medium [18]. In order to benefit more from the information contained in the diffused photons to access physical characterization of the medium, spatially resolved spectroscopy (SRS) was developed, based on NIR spectroscopy. In SRS, data is collected at different angles from the excitation light source in order to collect both the transmitted and diffused light. SRS has been successfully used in food [19], pharmaceutics [20] and chemical applications, like silica precipitation [21]. Emulsion polymerization consists of a heterogeneous medium that generates 
strong light diffusion related to particles, for which the SRS is well adapted. The aim of this work is therefore to determine the efficiency of monitoring the polymer content using SRS and to investigate the impact of each angle. Raman spectroscopy is also employed for comparison. Raman is not as sensitive as NIR to the physical characteristics of the medium and is therefore mainly sensitive to its chemical composition. The polymer content constitutes a good indicative of the progress of the reaction, as the monomer conversion can be calculated from it, which represents the first desired information in polymerization processes.

The paper is organized as follows: first of all, the SRS \& Raman spectroscopies are presented and regression models are developed to predict the polymer mass fraction for each method. Both methods are implemented for online monitoring in emulsion copolymerization of butyl acrylate and methyl methacrylate under different operating conditions. The advantages and drawbacks of the SRS and the Raman spectroscopy are discussed.

\section{Materials and method}

\subsection{Materials}

The used monomers are methyl methacrylate (MMA, Acros Organics, 99\%, stabilized) and butyl acrylate (BuA, Acros Organics, 99+\%, stabilized). Small amounts of acrylic acid (AA, Acros Organics, 98\% extra pure, stabilized) were added to enhance the nucleation and radical capture. The initiator was potassium persulfate (KPS, Acros Organics, 99+\%). The monomers and initiator were stored in a fridge until used. Sodium dodecyl sulfate (SDS, Fisher Chemical) was used as ionic surfactant. Deionized water of $18 \mathrm{M} \Omega \mathrm{cm}$ resistivity was used throughout the work.

\subsection{Ab initio semi-continuous polymerization experiments}

A $1 \mathrm{~L}$ reactor was used with mechanical stirring between $300-400 \mathrm{rpm}$ using a three blades Bohlender propeller. The reaction started by a batch polymerization period during which polymer particle nucleation took place, followed by a semi-continuous period to allow their growth. First, the SDS was dissolved in $800 \mathrm{~g}$ of water in the reactor and degassed using nitrogen for $30 \mathrm{~min}$ under stirring. The mixture was at the same time heated to $70{ }^{\circ} \mathrm{C}$ using a thermostated bath with silicon oil. Then, $40 \mathrm{~g}$ of a MMA/BuA/AA mixture was added and the polymerization was initiated by adding $1.6 \mathrm{~g}$ of KPS. During the reaction, the nitrogen gas flow was moved above the reaction medium, to the top of the reactor, to maintain saturation of the gaseous atmosphere with nitrogen. After particle nucleation, semi-continuous monomer addition was started by adding $160 \mathrm{~g}$ of the monomer mixture at a flow rate of $0.02 \mathrm{~g} / \mathrm{s}$. With this flow rate, starved conditions 
are achieved thus ensuring monomer consumption with no accumulations inside the reactor. Samples were collected at specific time intervals to measure the solids content (i.e. mass fraction of solid) using a thermobalance (Mettler Toledo L16) and the particles size distribution was measured using a dynamic light scattering (DLS, Malvern Nano ZS ${ }^{\circledR}$ ). The mass of surfactant and initiator were subtracted from the solid content to calculate the mass fraction of polymer.

\subsection{Design of experiments}

A design of experiments was done by choosing to vary different operating conditions, mainly the fraction of monomers, the amount of surfactant, the stirring rate and the impact of adding $A A$. The surfactant concentration was chosen in a way to start the reaction above the Critical Micellar Concentration (CMC, which is about $3.1 \mathrm{~g} / \mathrm{L}$ for SDS at $\left.70^{\circ} \mathrm{C}\right)$. In Table 1, the surfactant concentration is expressed in mass percentage according to the mass of monomer (MMA and BuA). The stirring rate was investigated to determine its effect on the quality of mixing and the measurement by the probes. The monomer composition was varied in a way to have the reaction temperature in some cases below and in others higher than the copolymer glass transition temperature $\left(\mathrm{T}_{\mathrm{g}}\right)$. This allows to detect different adhesion or sticking behaviors on the probes. Fox equation (1) was used to estimate the monomer composition in a way to have different glass transition temperatures and thus different polymer properties:

$$
\frac{1}{T_{g}}=\frac{\% M M A}{T_{g, M M A}}+\frac{\% B u A}{T_{g, B u A}}
$$

As a result, eleven runs were carried out with different operating conditions (Table 1). The first run is considered as a reference. The design of experiment was then built on small variations of parameters around this reference to evaluate how the spectroscopy is sensitive to these variations.

Table 1: Operating conditions of the different emulsion copolymerization runs

\begin{tabular}{cccccc} 
Run & $M M A(\%)$ & $B u A(\%)$ & $A A(\%)$ & $n(r p m)$ & Surfactant $(\% w t)$ \\
\hline $\mathbf{1}$ & $\mathbf{7 5}$ & $\mathbf{2 5}$ & $\mathbf{1}$ & $\mathbf{3 5 0}$ & $\mathbf{7 . 5}$ \\
2 & 75 & 25 & 1 & 300 & 5 \\
3 & 70 & 30 & 0 & 400 & 10 \\
$\mathbf{4}$ & $\mathbf{7 5}$ & $\mathbf{2 5}$ & $\mathbf{1}$ & $\mathbf{3 5 0}$ & $\mathbf{7 . 5}$ \\
5 & 70 & 30 & 0 & 300 & 10 \\
6 & 80 & 20 & 1 & 300 & 10 \\
7 & 75 & 25 & 0 & 400 & 5 \\
$\mathbf{8}$ & $\mathbf{7 5}$ & $\mathbf{2 5}$ & $\mathbf{1}$ & $\mathbf{3 5 0}$ & $\mathbf{7 . 5}$ \\
$\mathbf{9}$ & $\mathbf{7 5}$ & $\mathbf{2 5}$ & $\mathbf{1}$ & $\mathbf{3 5 0}$ & $\mathbf{7 . 5}$ \\
10 & 70 & 30 & 0 & 300 & 5 \\
11 & 70 & 30 & 1 & 400 & 5
\end{tabular}




\subsection{Raman spectroscopy}

A Raman spectrometer OceanOptics ${ }^{\circledR}$ (QE65000) was used for in situ measurements at an excitation wavelength of $785 \mathrm{~nm}$. The spectrometer is equipped with an Inphotonics immersion probe with a diameter of $9.5 \mathrm{~mm}$ and a working distance of $0.5 \mathrm{~mm}$. The integration time was set to 1 minute, with a boxcar width of 1 and an acquisition frequency of 1 spectrum per minute. The wavelength region covered was from $500 \mathrm{~cm}^{-1}$ to $3620 \mathrm{~cm}^{-1}$. The region before $500 \mathrm{~cm}^{-1}$ was eliminated to remove the Rayleigh scattering from the spectrum.

\subsection{SRS spectroscopy}

A multiangle probe Sam-Flex (Indatech ${ }^{\circledR}$, Chauvin Arnoux) was connected to a spectrometer from Indatech $^{\circledR}$ (Hyternity) which is composed of a NIR camera coupled with an InGaAs detector. The measurement was done simultaneously at four angles with a $3 \mathrm{~mm}$ pathlength. These angles consist of: one angle in the transmission direction, i.e. at $180^{\circ}$ (according to the light source), two angles in scattering directions at $170^{\circ}$ and $175^{\circ}$, and one angle in the backscattering direction at $30^{\circ}$ (Figure 1). The NIR spectral domain was from $871 \mathrm{~nm}$ to $1723 \mathrm{~nm}$, with a $3 \mathrm{~nm}$ interval, for each angle.

Physical attenuators were set manually and used at $180^{\circ}$ and $175^{\circ}$ in order to avoid saturation of the signal at this angle and still collect good signal at the other angles.

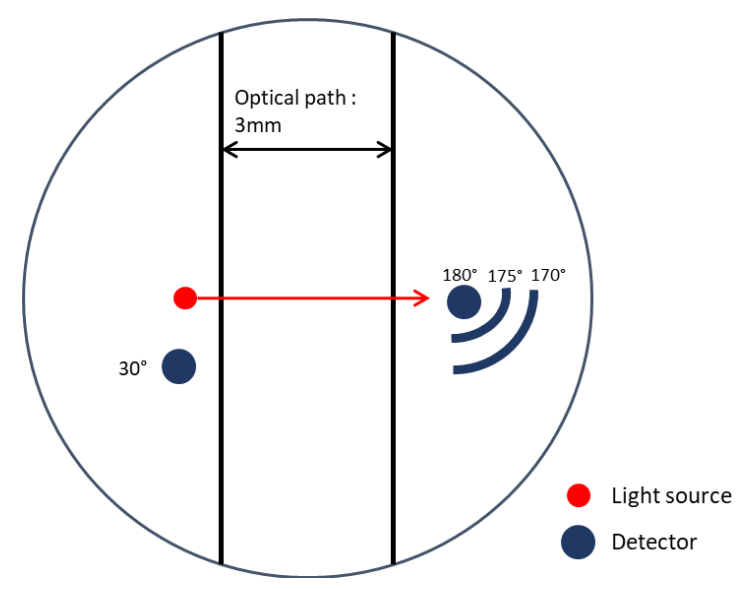

Figure 1: Measurement angles inside the SRS probe

\subsection{Chemometrics}

The chemometrics employed in this study are based on the partial least square (PLS) regression using the Nonlinear Iterative Partial Least Squares (NIPALS ) algorithm [22]. The PLS regression uses a combination of the different Raman shifts or wavenumbers in the NIR region and linearly 
correlates them to the polymer fraction in this work. All the chemometrics part was done using MATLAB R2017b ${ }^{\circledR}$.

\section{Results and discussion}

Figure 2 shows the evolution of the reaction rate, with the different samples, as a function of time in the different runs. At the beginning of the reaction, different reaction rates are observed because of the different compositions in monomers. However, when the semi-batch period starts, the reaction rate of the different runs is similar because the reaction is done under starved conditions, and therefore the reaction rate is determined by the monomer addition flowrate. The observed fluctuations in this range are possibly caused by the variations of the pump used for the introduction of monomers. It is required to predict the effect of the changes in the different parameters on the reaction, or on the monomer conversion, which have to be monitored by the in situ probes. The progress of the reaction can be determined by monitoring the concentration of residual monomer or the quantity of formed polymer.

Figure 3 shows the number of particles per liter (measured by NanozS) as a function of the polymer fraction for the different runs. It can be seen that the main parameter affecting the number of particles is the concentration of surfactant. As expected, a higher surfactant concentration leads to the formation of a bigger number of particles. It is hard to detect an impact of the presence of acrylic acid on the monomer conversion or the number of particles in these experiments, probably due to its low concentration. Similarly, the effects of the monomer composition and the stirring rates on the number of particles are negligible in the investigated ranges. 


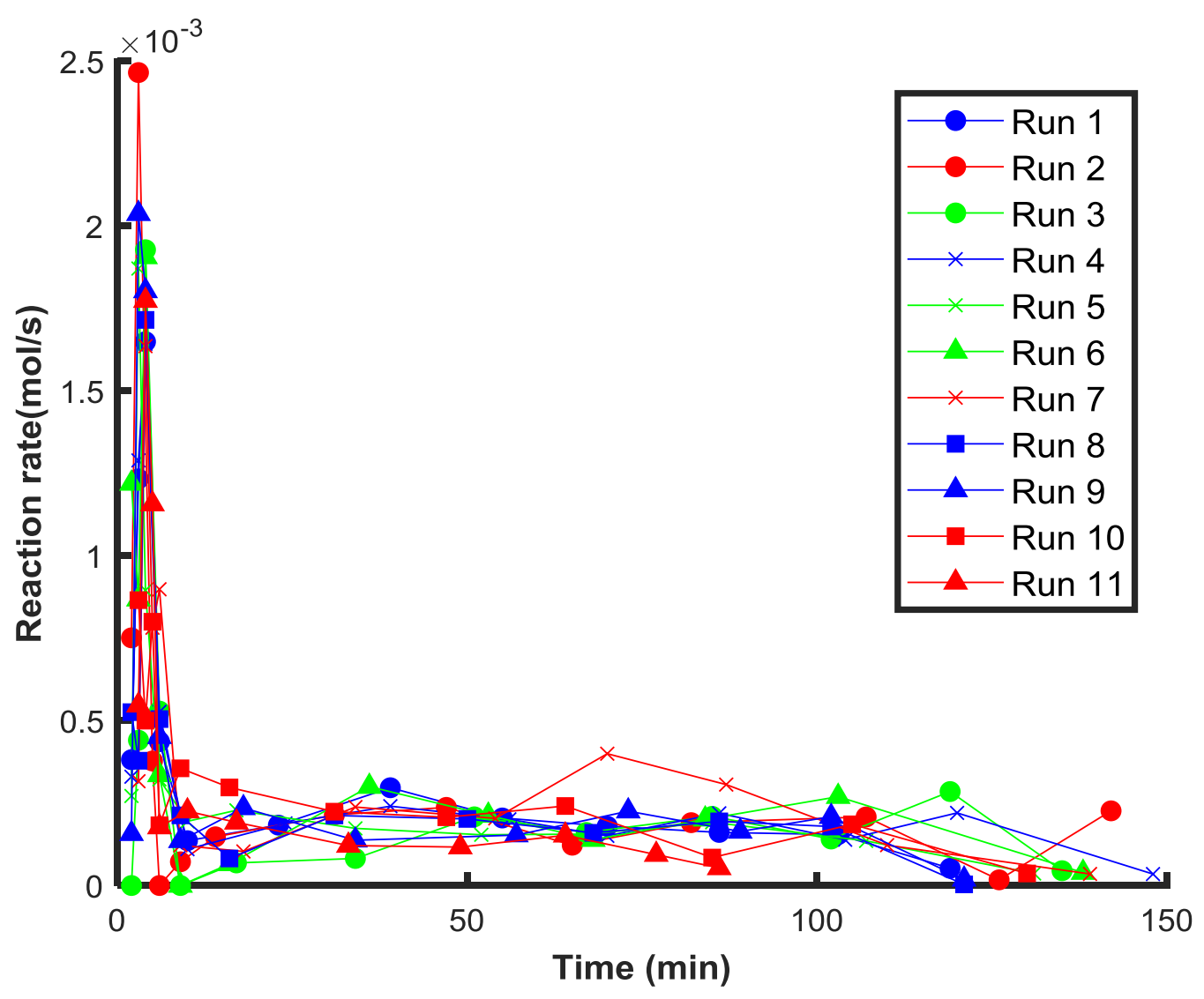

Figure 2: Reaction rate as a function of time for the different runs 


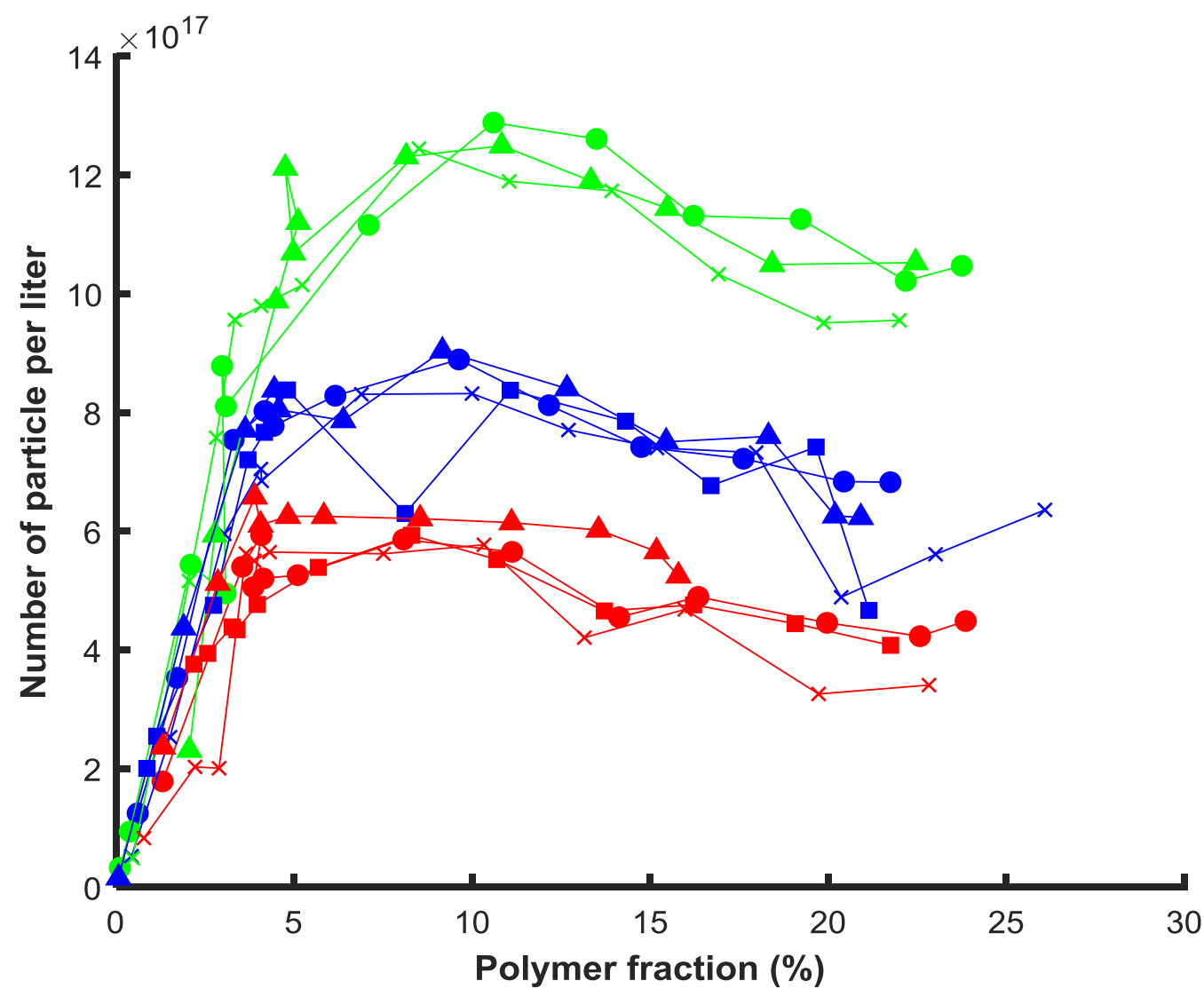

Figure 3: Number of particles per liter as a function of the mass polymer content for the different runs

Online monitoring has to deal with the varying process conditions of temperature, aggressive monomer, mixing and fouling. In the case of predicting the polymer fraction, it is essential to overcome the difficulties due to the reaction heterogeneity and decouple it from changes in the particle size and number. In the following, both the Raman and SRS spectroscopies are introduced and employed to monitor this system.

\subsection{Raman spectroscopy}

In these experiments, the polymer content varies between 0 and $25 \%$ wt. The SRS probe was subject to fouling which did not allow to monitor well the polymer content at higher values. At the beginning of the batch period, the reaction rate is very high, and the polymer fraction varies between 0 and $5 \%$ wt in a very short time period. The optimal acquisition time of the Raman spectrometer that ensured a compromise between a sufficiently high intensity without saturating the signal, was found to be $1 \mathrm{~min}$. Due to this long acquisition time, the signal was found not to be representative of the reaction medium in the initial reaction period. The Raman spectra were thus treated only in the range of 5-25\% wt of polymer fraction, so mainly in the semi-batch period. 
Figure 4 shows the Raman spectra acquired every minute during the semi-batch period. The spectra are colored according to time, going from blue $(t=0)$ to red. It can be seen that the whole spectrum evolves with time. The important chemical functions to be investigated here are the C$\mathrm{O}-\mathrm{C}$ at $836 \mathrm{~cm}^{-1}$, the $\mathrm{C}=\mathrm{C}$ at $1657 \mathrm{~cm}^{-1}$, the $\mathrm{C}=\mathrm{O}$ at $1751 \mathrm{~cm}^{-1}$ and the $-\mathrm{CH}_{2}$ at $2960 \mathrm{~cm}^{-1}$. The $\mathrm{C}=\mathrm{C}$ bound is characteristic of the residual monomers. The intensity of the band correlated to this bound evolves by two ways: a decrease with time because the monomer is converted into polymer, and an increase due to the continuous addition of monomer (semi-batch operation). Due to the low concentration of monomer compared to the increasing amount of polymer, and the presence of most monomer within the polymer particles, monitoring the $\mathrm{C}=\mathrm{C}$ bound by $\mathrm{a}$ univariate treatment has proven to be inaccurate and noisy and will not be shown here. The $-\mathrm{CH}_{2}$ bound is characteristic of the polymer formed during the reaction. However, this $-\mathrm{CH}_{2}$ bound is also present in the monomers, and therefore univariate treatment based on this band is not appropriate to predict the polymer fraction in this study. A multivariate treatment is therefore necessary.

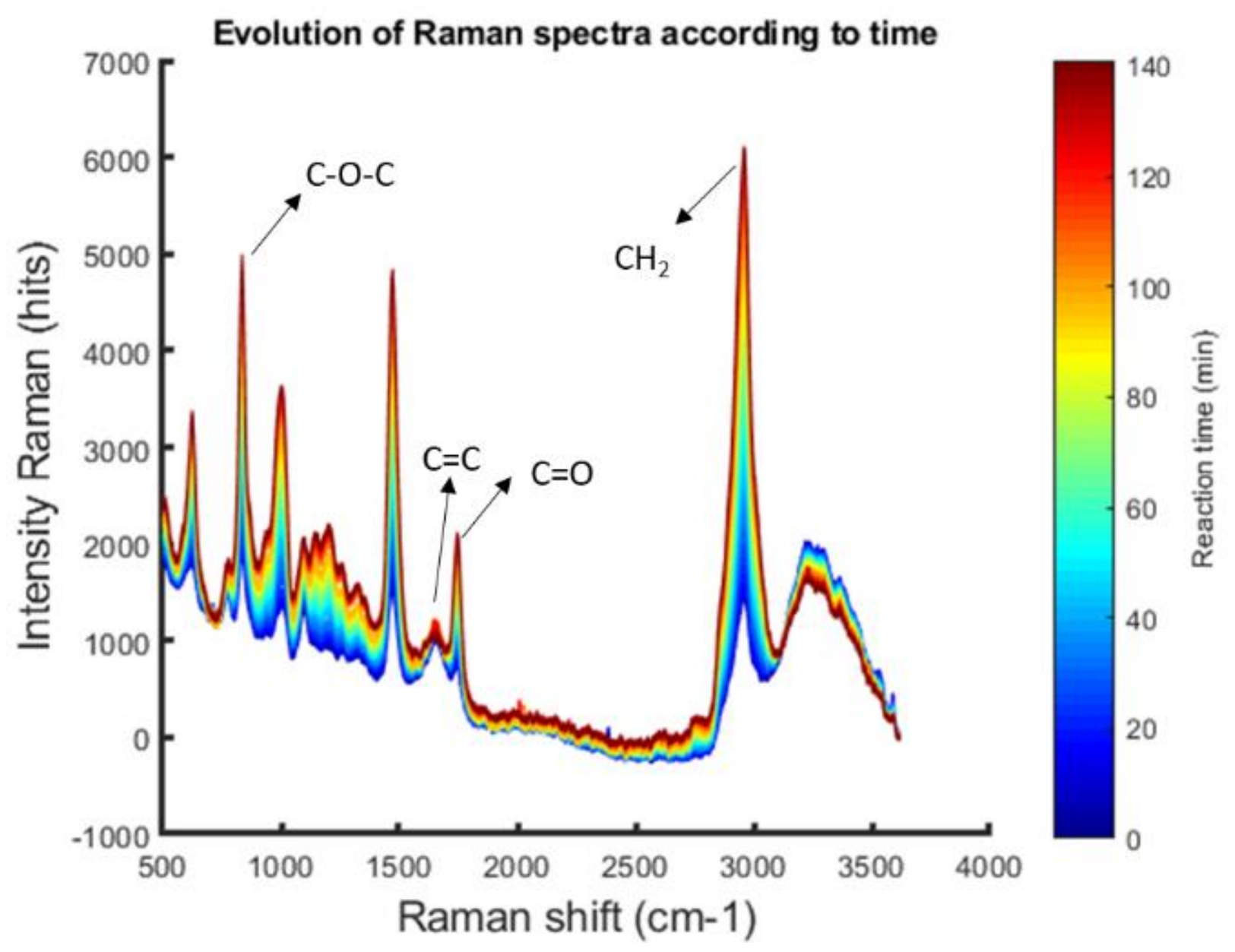

Figure 4: Evolution of the Raman raw spectra according to time for run 6 
The Partial Least Square (PLS) regression was thus used to develop a multivariate prediction model. The spectral ranges selected for this regression were $500-1781 \mathrm{~cm}^{-1}$ and $2794-3620 \mathrm{~cm}^{-1}$. No significant chemical band is present on the spectra in the area between these two regions, and therefore there is no need to include it. The used set of data was pretreated with a Savitzky-Golay algorithm [23] (15 points averaged and polynomial of second order for smoothing, and first order derivative). A total of 66 spectra were used. 44 spectra were used to develop the calibration model and the 22 remaining spectra were used for model validation. The selection was done by regrouping all the runs in a matrix, and 1 spectrum out of 3 was taken for model validation. The remaining spectra were kept for the development of the calibration model. Runs 2 and 7 were not used in the Raman model because many fluctuations were observed in the acquired Raman spectra. This can be due to the position of the probe in the reactor (e.g. relative to the agitator or to the monomer feed) that could not be maintained identical in all runs due to the opacity inside the reactor.

In order to determine the optimal number of latent variables (LVs, i.e. the number of components for the regression), the root mean square error of calibration (RMSEC) and prediction (RMSEP)were plotted against the number of LVs (Figure 5). The optimal number of latent variables is the one giving the lowest RMSEP and RSMEC in order to avoid overfitting and provide at the same time ensure robustness of the model. Figure 5 shows that the minimal RMSEC and RMSEP are achieved with 4 LVs.

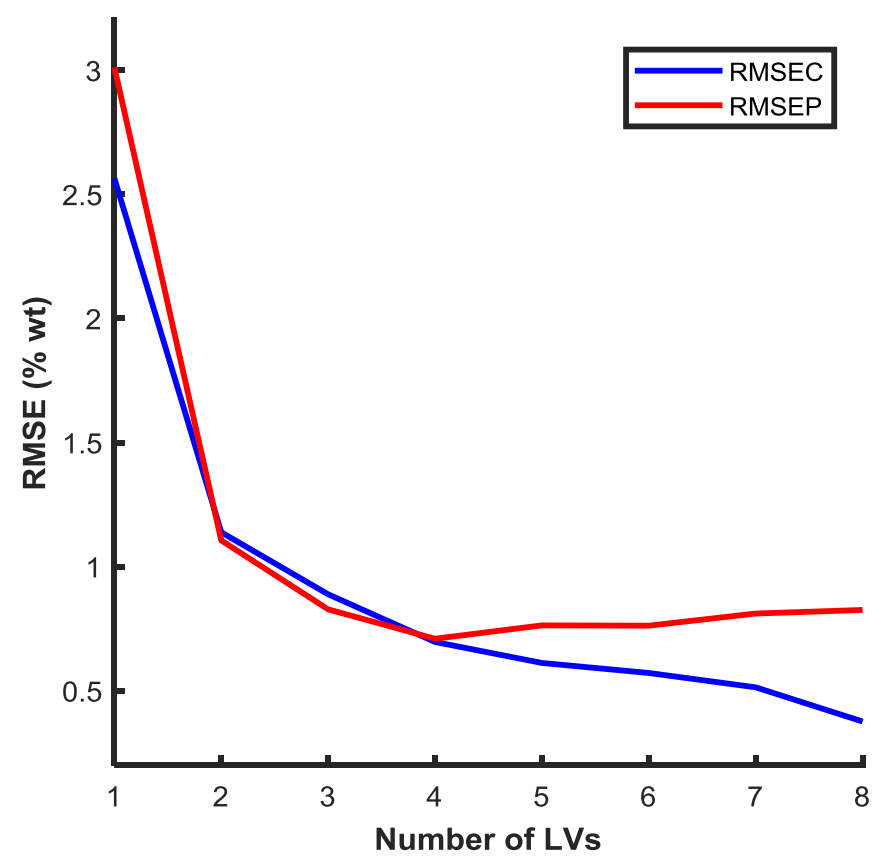


Figure 5: Root Mean Square Error of Calibration and Prediction according to the number of latent variables using the PLS model applied to Raman data

The validity of the model predictions of the polymer content for both the calibration and prediction samples is studied by investigating the residuals. The residuals are the differences between the predicted and the experimental values. Figure 6 shows the residuals of the different data from calibration and validation. The acceptable limit, represented with the pink dots, is defined by taking a confidence interval of $95 \%$ (twice the RMSEC). The calibration and prediction errors, were calculated respectively at $\mathrm{RMSEC}=0.70 \%$ wt and $\mathrm{RMSEP}=0.71 \%$ wt. The residuals show that the model predictions using the Raman spectroscopy are in good agreement with the references obtained by gravimetry.

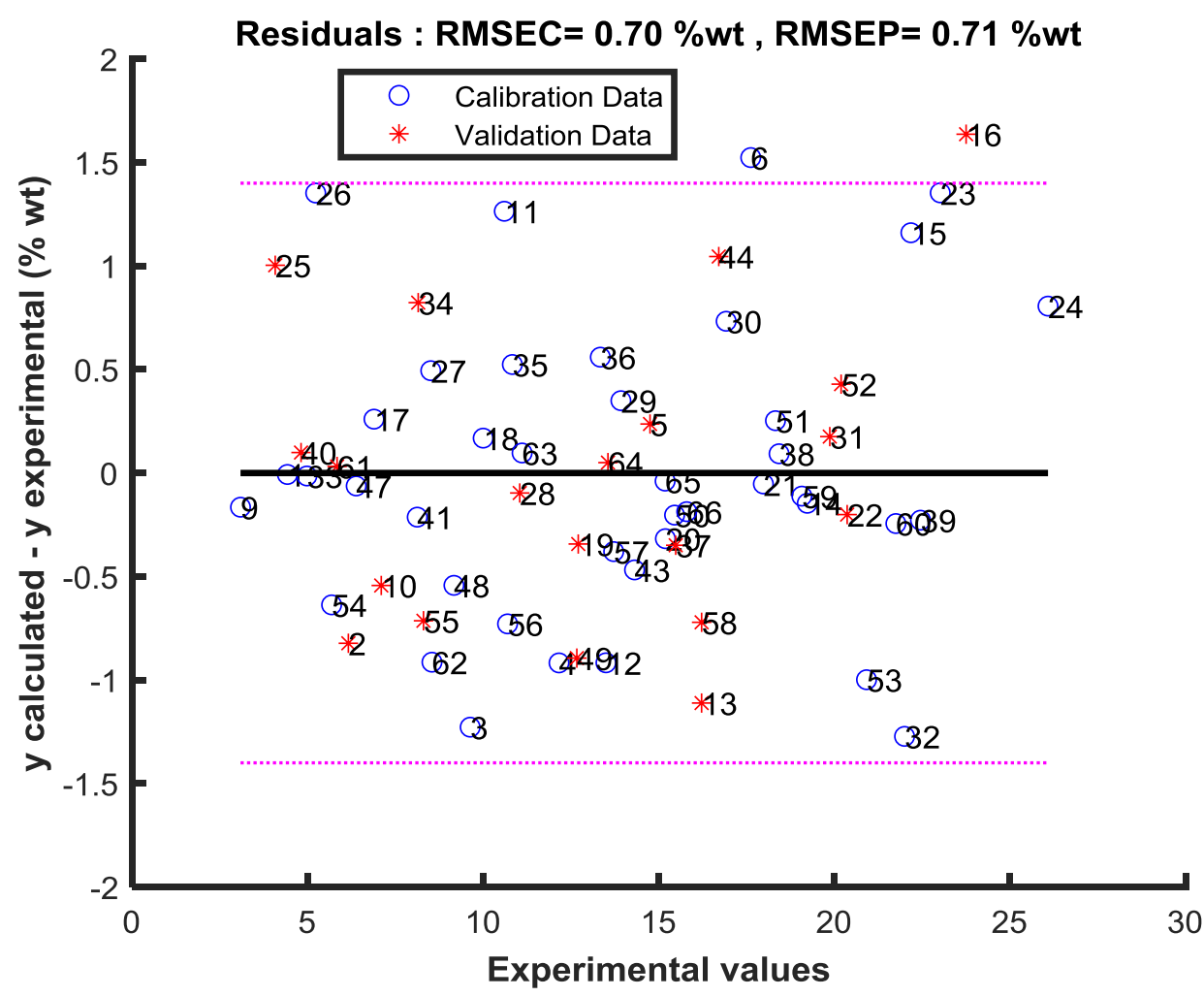

Figure 6: Residuals of the different data points predicted using PLS regression applied to Raman data

The uncertainty of the model developed in this work (RMSEC $=0.70 \%$ ) was found to be in agreement with the literature, with some improvements. For instance, in the work of Elizalde et al., an RMSECV around $0.9 \%$ wt was reached using online Raman spectroscopy in a similar emulsion polymerization process [13].

The established regression model was then used to investigate the influence of the varied parameters in the different runs on the evolution of the reaction using the online Raman data 
(Figure 7). The effect of the agitation speed can be examined by comparing runs 3 and 5, with the agitation speeds of $400 \mathrm{rpm}$ and $300 \mathrm{rpm}$ respectively (Figure 7a). Both experiments behave similarly as shown by offline gravimetry as well as online Raman spectroscopy predictions which indicates that there is no effect of the agitation speed on the uniformity of the medium, nor on the coagulation of particles. A small fluctuation of the predictions by Raman is observed in the experiment with an agitation speed of $400 \mathrm{rpm}$. This could be due to the closeness of the probe to the agitator, but not to the increase in the agitation speed, as fluctuations were not observed in other experiments realized at $400 \mathrm{rpm}$.

Concerning the quality of the regression model, the best performance is obtained for the intermediate polymer content. The spectra at the beginning of the reaction (i.e. for low polymer content) were not treated due to the fast reaction rate which would require a different acquisition time as explained above. Toward the end of the reaction, the model predictions are less accurate for high polymer fractions which can be explained by the fact that only few points in the learning data contain high polymer fractions. It would therefore be helpful to complete the calibration data base with higher fractions of polymer.

Figure $7 \mathrm{~b}$ shows the prediction of runs 5 and 6 for which only the MMA (and so BuA) weight fraction (compared to the total mass of monomers) changed from $70 \%$ to $80 \%$. At $70 \%$ wt of MMA, the prediction with Raman tends to be slightly underestimated at low polymer content then overestimated at higher polymer content but the trend is preserved and remains acceptable. During the batch period, a higher fraction of MMA led to a higher reaction rate, which should be due to its higher solubility in water (The solubility of MMA in water is much higher than BuA, $\phi_{M M A}^{w}=0.15 \mathrm{~mol} . \mathrm{L}^{-1}$ and $\phi_{B u A}^{w}=0.01 \mathrm{~mol}^{-\mathrm{L}^{-1}}$ at $45^{\circ} \mathrm{C}$ [24]) which enhances the propagation of primary radicals and their capture by the micelles and particles. This cannot be confirmed with Raman data as batch period cannot be exploited. During the semi-continuous period, changing the monomer composition from $70 / 30$ to $80 / 20$ does not lead to a significant change of the reaction rate. This is explained by the fact that monomer addition is done under starved conditions, therefore both monomers are consumed gradually without accumulation of either monomer. Note that the monomer addition was not started exactly at the same time for all the experiments, which may create a difference in the polymer fraction with time.

Figure 7c shows the prediction of runs 4, 6 and 10 for which the surfactant concentration is $7.5 \%$ wt, $10 \%$ wt and $5 \%$ wt respectively. As described previously (Figure 3), the number of particles 
was increasing with the surfactant concentration, which led to a slightly higher reaction rate during the batch period. Thereafter, a good prediction of the conversion can be observed.

The studied parameters thus validate the robustness of the Raman model of prediction in terms of the various operating conditions with a RMSEC close to $0.7 \% \mathrm{wt}$ of polymer. In the next section, the SRS is employed for the same experiments in order to evaluate its potential.
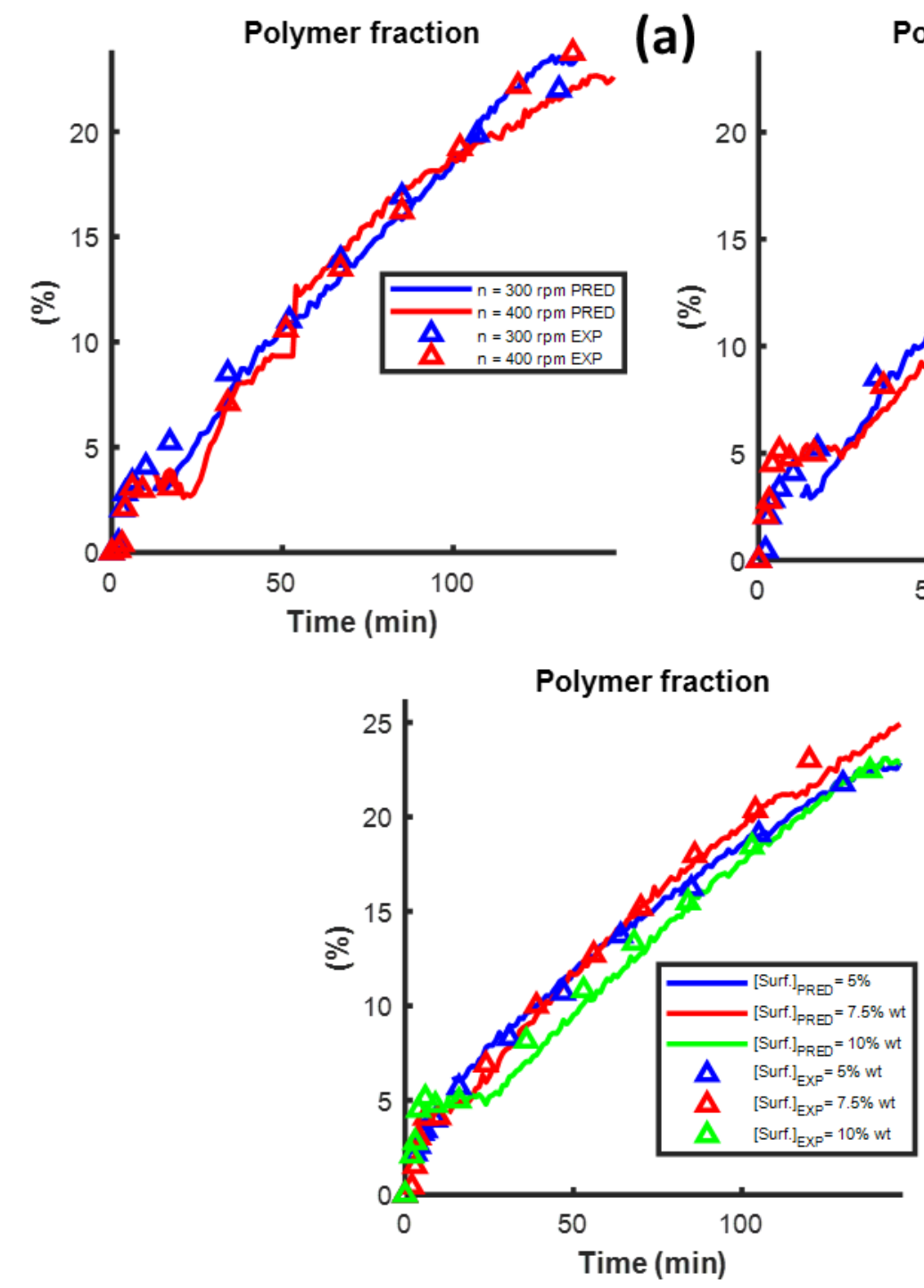

(a)

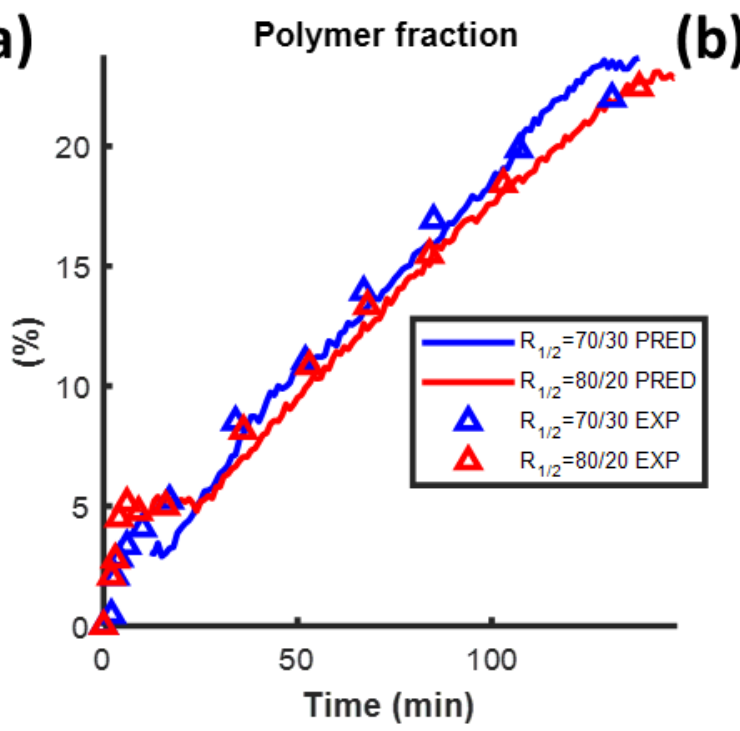

(c)

Figure 7 : Prediction of the polymer fraction by online Raman spectroscopy, for varying (a) agitation speed, (b) monomer composition, and (c) surfactant mass fraction

\subsection{Spatially resolved spectroscopy (SRS)}

Spatially resolved spectra were collected at the angles of $180^{\circ}, 175^{\circ}, 170^{\circ}$ and $30^{\circ}$ in the NIR region. However, the data collected in the transmission direction, i.e. at $180^{\circ}$, could not be exploited in this study because the signal-to-noise ratio was too low. Note that manual 
attenuators were implemented in order to avoid saturation of the receptors by the signal. However, this manual attenuation was too strong at the beginning of the reaction in the absence of polymer in order to scatter the incoming beam. But, once the reaction started, the medium became quickly opaque and the transmission signal decreased drastically. The bands in transmission were still noticeable but the noise complicated the data treatment.

The acquisition time of SRS was much shorter than Raman, $15 \mathrm{~s}$ for SRS against 1 min for Raman. Therefore, the batch period could be investigated using the SRS to estimate the polymer fractions starting from 0 to $25 \%$ wt. However, in order to allow comparison between the SRS and the Raman, the model was developed based on data in the range 5-25\% wt. Only the prediction was done on the range $0-25 \% \mathrm{wt}$. Figure 8 shows the SRS spectra collected at the angles $175^{\circ}, 170^{\circ}$ and $30^{\circ}$ every $15 \mathrm{~s}$ during the reaction (from blue to red). The spectra were put one after the other, in an unfolded way, with each spectrum acquired in the range $871-1723 \mathrm{~nm}$. The spectral intensity corresponds to the light received by the camera. All spectra evolve during time but, as expected from the NIR spectra, it contains no direct correlations to chemical structures, that can be interpreted directly, as it exhibits overtones and combinations of fundamental vibrations of infrared. The multivariate analysis is therefore required in order to develop a prediction model of the polymer content, as traditionally done for NIR data [27]-[29]. 


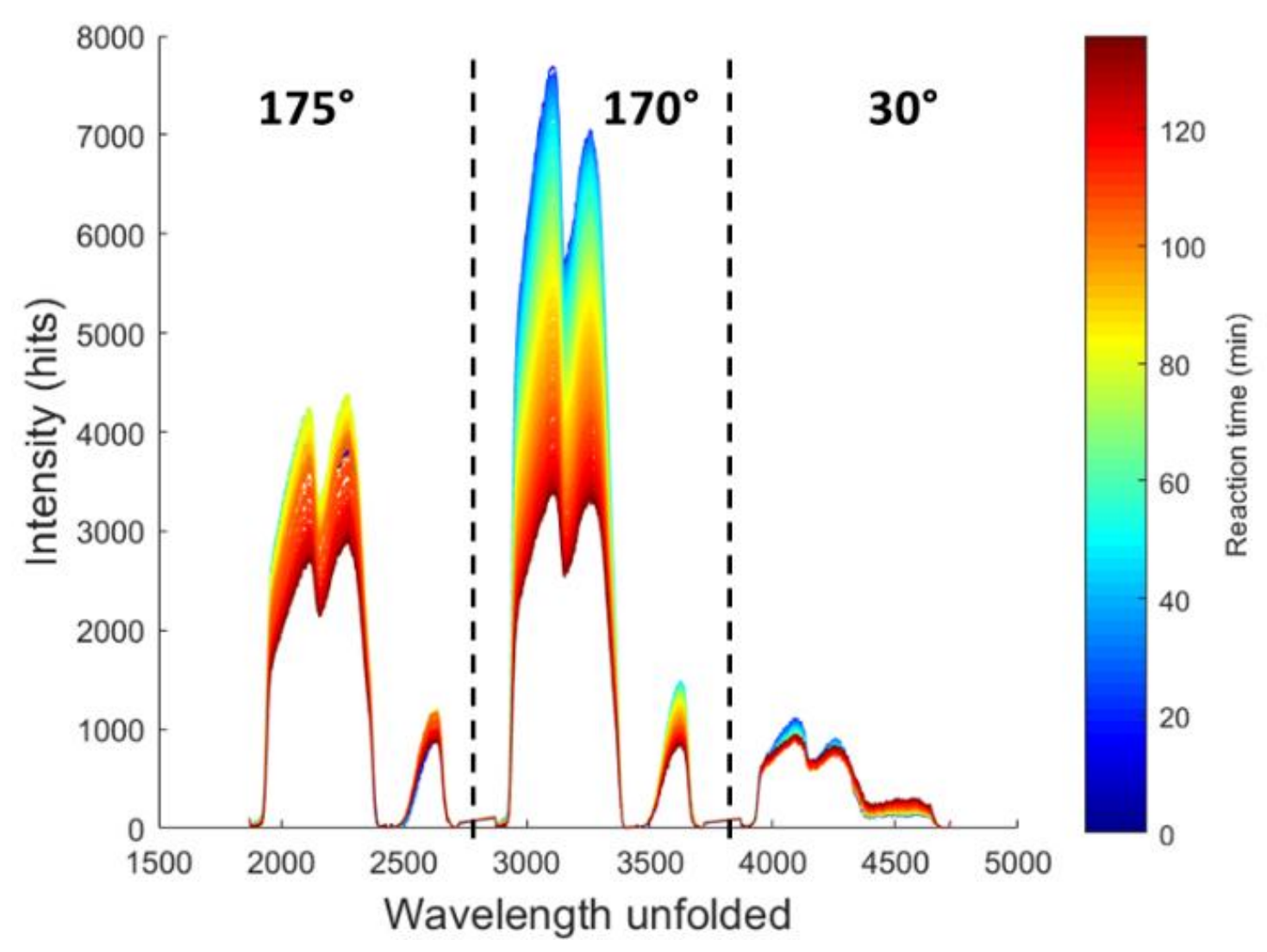

Figure 8: SRS spectra at angles $175^{\circ}, 170^{\circ}$ and $30^{\circ}$, collected during run 6 , according to reaction time

Runs from 8 to 11 were not used because important fluctuations were observed in the SRS spectra with time. This can be due to the position of the probe (close to the agitator or monomer introduction), the presence of bubbles into the probe, or a stability issue of the optical connections of the attenuators. As a reminder, for Raman implementation, runs 2 and 7 were rejected due to instability of data. Also note that the Raman probe is flat while the SRS probe has a cavity, therefore bubbles are more likely to get stuck into the SRS cavity than on the Raman probe. A total of 48 spectra were used, with 32 of them for the development of a calibration model and the 16 remaining spectra for model validation. The selection was done on a similar base as for Raman.

The choice of the number of latent variables is done based on the RMSEC and RMSEP, as done previously for the Raman data. Figure 9 shows the RMSEC and RMSEP as a function of the number of LVs, where both minima are reached with 4 to 7 LVs. In this case, 5 LVs were chosen. 


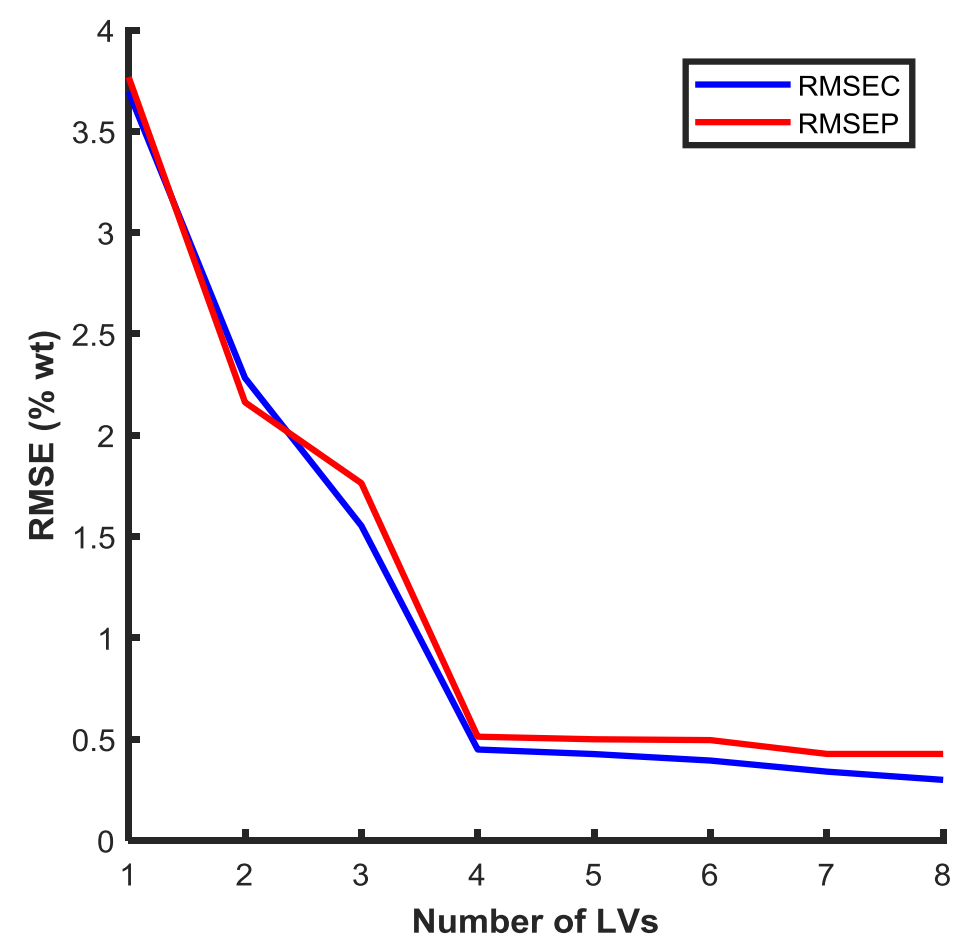

Figure 9 : Root Mean Square Error of Calibration and Prediction according to the number of latent values using the PLS model implement on the SRS data

Gossen \& Al.[16] obtained a model with a standard error of prediction around $0.5 \%$ wt using classic NIR spectroscopy. Figure 10 shows the residuals of the polymer fraction prediction using the SRS data. The calibration and validation data are both almost included within the interval of confidence of $95 \%$ (twice the RMSEC). The calibration error is around $0.43 \% \mathrm{wt}$ and the prediction error is around $0.50 \% \mathrm{wt}$, so similar to classic NIR spectroscopy obtained in the literature. This limit is better than that obtained by the Raman predictions. Note that classic NIR spectroscopy is usually obtained from transmission at $180^{\circ}$, or by back scattering at $0^{\circ}$. The SRS spectrum, without the angle at $180^{\circ}$, does not include direct transmission information. However, the information is still contained with the other angles as photons are necessarily transmitted in multiple pathlengths before being deflected making the transmission information partially present. Therefore, even in the absence of the direct transmission information at $180^{\circ}$, the model predictions by the SRS can be equivalent to classic NIR and the addition of this information would probably provide a better model. 


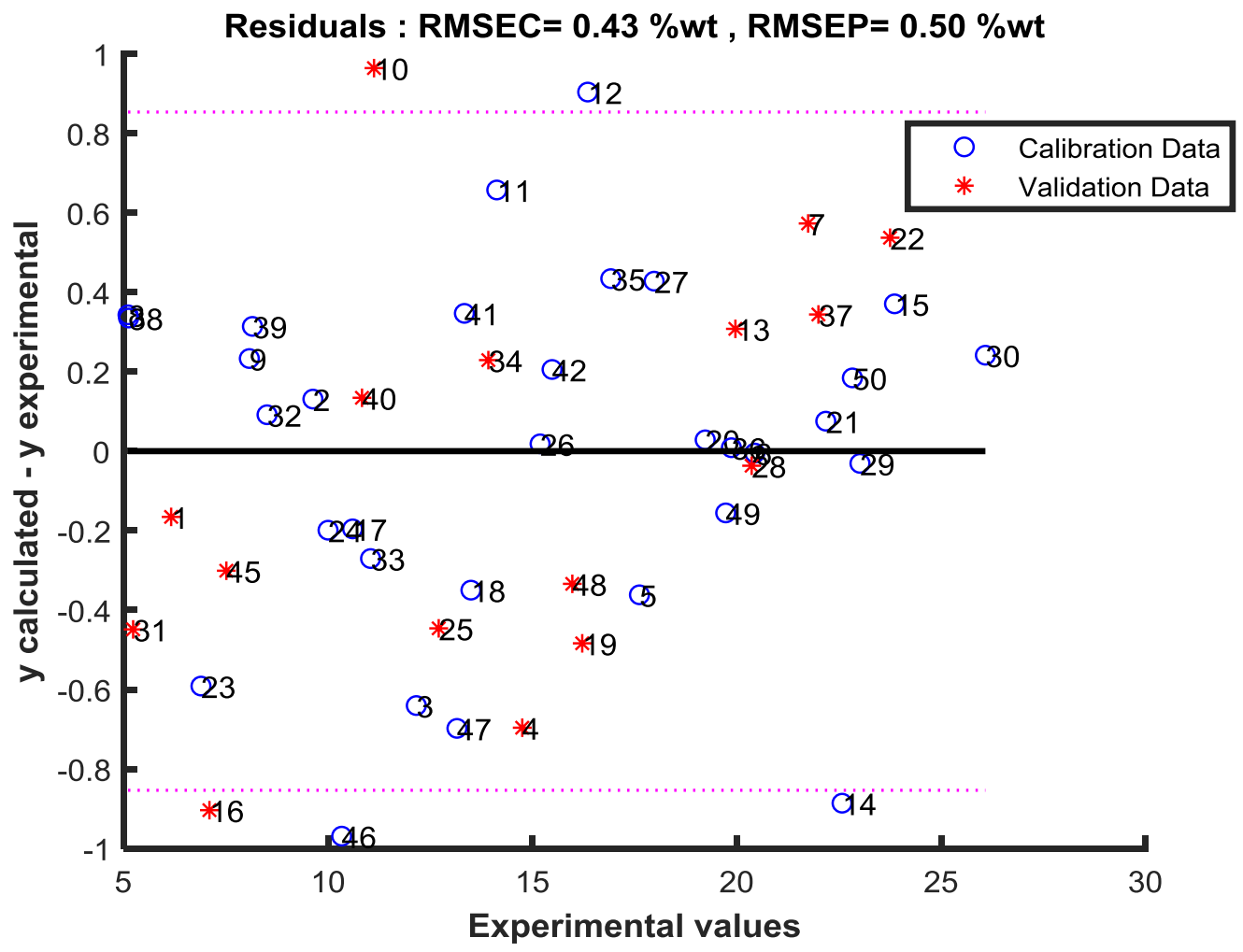

Figure 10: Residuals of the different data points of the polymer mass fraction predicted using PLS regression implemented on the SRS data

The influence of the different process parameters could then be investigated using the SRS predictions. Figure 11a shows the results of runs 3 and 5 with an agitation speed of 400 and 300 rpm respectively. It was concluded above, based on gravimetry and particle size measurements, that the stirring rate in the investigated region does not have any effect on the reaction rate nor on the product properties. Figure $11 \mathrm{a}$ also indicates that the stirring rate does not influence the performance of the probe in this region. A higher precision is obtained using the SRS compared to the Raman spectra for these two experiments, as both experiments follow exactly the same trend without any fluctuation. This confirms that the fluctuations observed in run 3 by the Raman probe are not due to a change in the reaction medium but only to a phenomenon correlated to the Raman probe and its position in that experiment. Note that the predictions of the SRS are good also in the high polymer fraction region, while a bias was systematically obtained in this region using the Raman spectra.

Figure $11 \mathrm{~b}$ shows the predictions of runs 5 and 6 where the MMA weight fraction total amount of monomer was $70 \%$ and $80 \%$ respectively. A good prediction of the polymer content is obtained for both experiments by the SRS-based model. The SRS predictions indicate a faster reaction with 
the higher fraction of BuA. Only in run 6 (with MMA weight fraction of $80 \%$ ) a significant bias is observed at the end of the reaction. This could be due to latex fouling in the gap of the SRS probe that was observed at high solid content. Note that the Raman probe is flat and does not contain any gap, and would possibly be less prone to fouling.

Figure $11 \mathrm{c}$ shows the predictions of runs 1,2 and 4 with surfactant mass fraction of $7.5 \%$ wt, for runs 1 and 4 , and $5 \%$ wt for run 2 . A good prediction is obtained for all surfactant mass fractions and no impact of the surfactant fraction, which is correlated to the number of particles (cf. Figure $3)$, is observed.
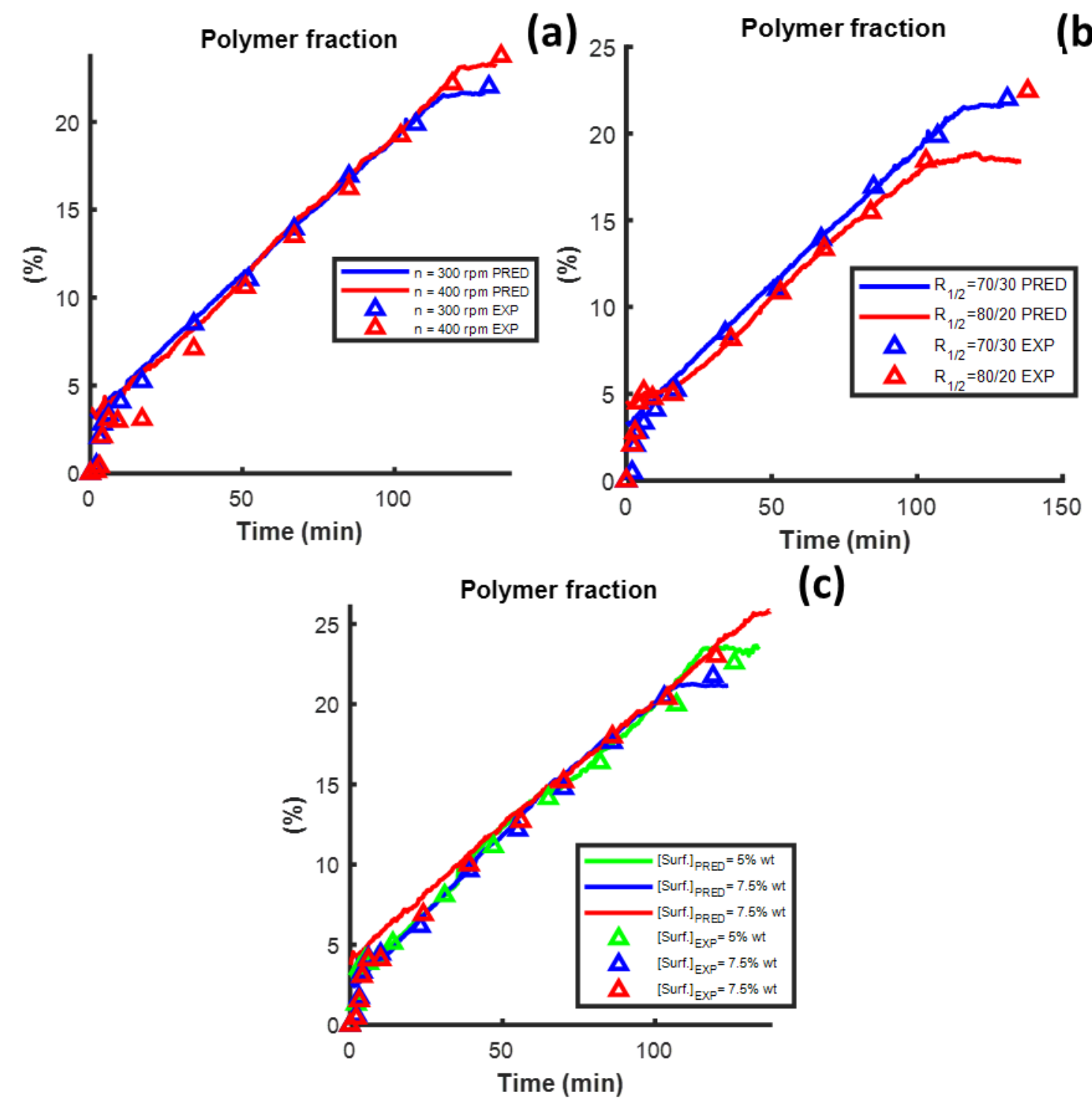

Figure 11: Prediction of the polymer content using the SRS, for varying (a) agitation speed, (b) monomer composition, and (c) surfactant mass fraction. 


\subsection{Comparison of Raman and SRS}

Different conclusions can be drawn when comparing the predictions of the polymer fraction by the Raman and SRS spectroscopies. Figure 12 shows the predictions of runs 1, 3, 4, 5 and 6 that were monitored and treated by Raman and SRS.

In general, a greater accuracy is obtained using the SRS for all runs, except at the end of run 6 as discussed previously. Also, the SRS presents a more stable signal and less fluctuations with time than the Raman. For instance, the results of run 3 are much less accurate using Raman than using SRS, and the fluctuations observed in the Raman spectra are not representative of the process, as they do not appear in the gravimetry or particle size measurements. Also, with the Raman spectrometer used here, the optimal integration time was one minute, which did not allow us to monitor efficiently the initial batch period, where the reaction rate is very high and averaging the spectra over one minute is not adapted. Furthermore, the models could not be established with reasonable error for the initial 2 minutes due to the lack of reference data in this period. Even with the absence of reference data, the SRS could predict well the polymer mass fraction in the nucleation period.

However, the SRS probe showed more difficulties related to the presence of air bubbles that were difficult to remove. This made a portion of the runs useless. Installing the SRS probe on an incline in the reactor would probably prevent bubbles from sticking in the measurement gap. For the Raman, no air bubbles stayed stuck on the probe as it is flat. Raman spectroscopy could therefore be used more systematically, though with a lower precision. Using a Raman spectrometer with a higher signal to noise ratio (or resolution) would certainly improve the prediction quality.

It is reasonable to suppose that combining the Raman and SRS data to build a calibration model would provide a better prediction accuracy. Merging the data sets should be done in an appropriate manner to be efficient. However, in this system, no real gain in terms of predictive capability was obtained. Instead of combining the benefits of the two techniques, the combined prediction accumulated the problems met with the two making the system less robust. Furthermore, from a practical point of view, combining the two techniques for process monitoring would constitute an expensive monitoring solution. 

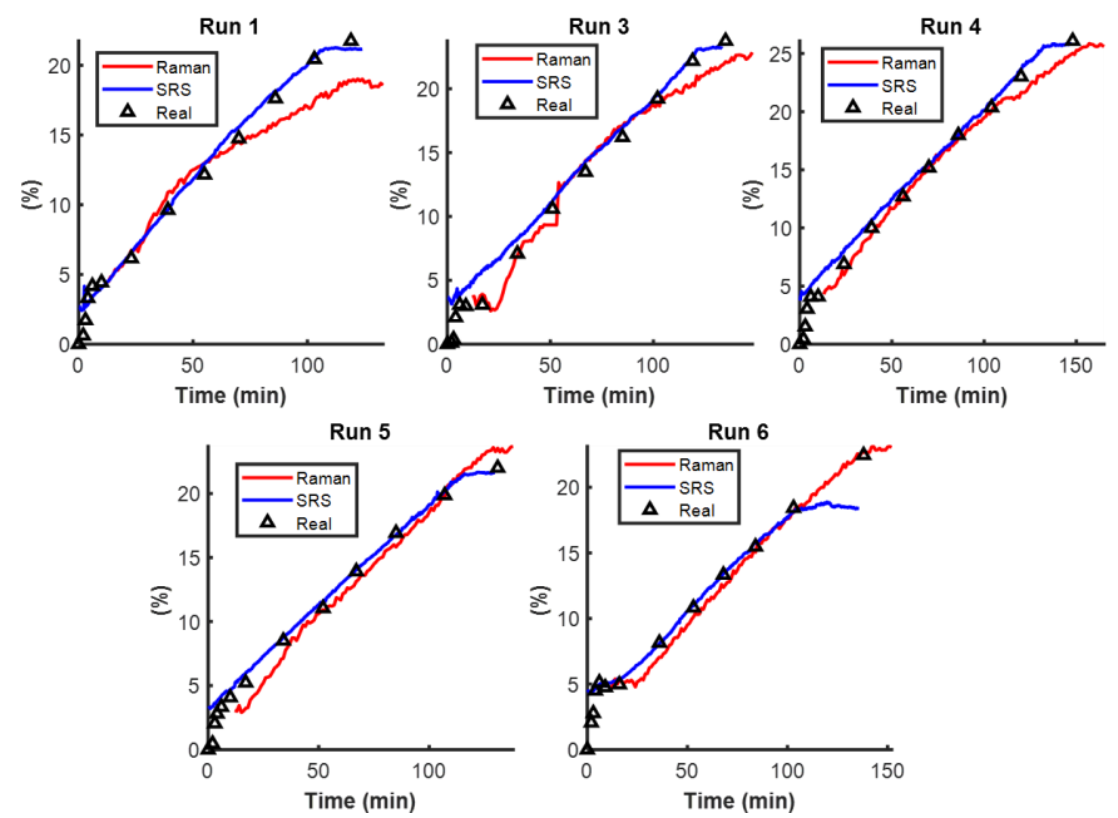

Figure 12: Predictions of the polymer fraction for runs 1, 3, 4, 5 and 6 using SRS and Raman spectroscopy

\section{Conclusions}

The use of spatially resolved spectroscopy was evaluated in emulsion copolymerization of MMA and BuA and the results were compared to Raman spectroscopy. It is shown that the prediction of the polymer fraction by SRS provides at least similar results as classic NIR, even though the direct transmission information at $180^{\circ}$ could not be used in this work. Also, the SRS generally provided better predictions compared to Raman spectroscopy. The prediction was robust to changes in the agitation speed and could detect small changes in the evolution of the polymer fraction when changing the MMA/BuA ratio. In the investigated scope, as the reaction takes place under starved conditions, the surfactant mass fraction had little impact on the reaction rate in the semicontinuous period, where the monomer feed becomes the limiting factor.

The data used for the models contains polymer fractions up to $20 \%$. The Raman model was however less efficient at higher polymer fractions. It would be good to include data with much higher polymer fractions to make the model more precise over a wider range of variation.

This work demonstrates the potential of the SRS for online monitoring of emulsion polymerization. It is highlighted that the geometry of the probe may lead to bubbles getting stuck within the measurement gap. Therefore, the positioning in a process loop should be well thought of. Latex fouling into the probe at high polymer fractions is also a risk, so proper cleaning protocols should be established for industrial online applications. In order to solve this problem of attenuation at 
the transmission angle, the possibility of using automated attenuators is developed by the manufacturers of the SRS spectrometer.

The interest of the SRS is to obtain both chemical and physical information at the same time. The capacity to monitor chemical properties has been demonstrated in this paper. The case of physical information, like the diameter of particles, will be detailed in another paper.

Acknowledgment: This work was funded by the pole Lyon Process Science and Engineering, LPSE.

\section{References}

[1] A. D. Trafford, R. D. Jee, A. C. Moffat, et P. Graham, " A rapid quantitative assay of intact paracetamol tablets by reflectance near-infrared spectroscopy », p. 5, 1999.

[2] P. A. Hailey, P. Doherty, P. Tapsell, T. Oliver, et P. K. Aldridge, « Automated system for the online monitoring of powder blending processes using near-infrared spectroscopy part I. System development and control », J. Pharm. Biomed. Anal., vol. 14, n 5, p. 551- 559, mars 1996.

[3] G. Fevotte, "New Perspectives for the Online Monitoring of Pharmaceutical Crystallization Processes Using in situ Infrared Spectroscopy ", ChemInform, vol. 34, n² 23, juin 2003.

[4] E. Gulari, K. McKeigue, et K. Y. S. Ng, " Raman and FTIR spectroscopy of polymerization: bulk polymerization of methyl methacrylate and styrene ", Macromolecules, vol. 17, $\mathrm{n}^{\circ}$ 9, $\mathrm{p}$. 1822- 1825, sept. 1984.

[5] F. H. Florenzano, P. Enohnyaket, V. Fleming, et W. F. Reed, "Coupling of near infrared spectroscopy to automatic continuous online monitoring of polymerization reactions ", Eur. Polym. J., vol. 41, n 3, p. 535- 545, mars 2005.

[6] T. Genkawa, M. Watari, T. Nishii, et Y. Ozaki, « Development of a Near-Infrared/Mid-Infrared Dual-Region Spectrometer for Online Process Analysis ॥, Appl. Spectrosc., vol. 66, $\mathrm{n}^{\circ}$ 7, p. 773- 781, juill. 2012.

[7] L. M. Gugliotta, M. Arotçarena, J. R. Leiza, et J. M. Asua, « Estimation of conversion and copolymer composition in semicontinuous emulsion polymerization using calorimetric data ", Polymer, vol. 36, nº 10, p. 2019- 2023, mai 1995.

[8] I. S. de Buruaga, A. Echevarría, P. D. Armitage, J. C. de la Cal, J. R. Leiza, et J. M. Asua, " Online control of a semibatch emulsion polymerization reactor based on calorimetry ", AlChE J., vol. 43, n 4, p. 1069- 1081, avr. 1997.

[9] L. V. de la Rosa, E. D. Sudol, M. S. El-Aasser, et A. Klein, "Details of the emulsion polymerization of styrene using a reaction calorimeter ", J. Polym. Sci. Part Polym. Chem., vol. 34, $n^{\circ} 3$, p. 461- 473, 1996.

[10] F. Farshchi Tabrizi et M. M. Fadaee, « Experimental investigation of continuous conductivity measurements during emulsion polymerizations having unstable/high-fouling reaction mixture ", J. Appl. Polym. Sci., vol. 134, nº 6, févr. 2017.

[11] B. Colegrove et al., "Use of Online Spectroscopy to Control Polymerization in Industrial Processes ", Macromol. React. Eng., vol. 11, n 4, p. 1600056, août 2017.

[12] R. A. M. Vieira, C. Sayer, E. L. Lima, et J. C. Pinto, " In-line andin situ monitoring of semi-batch emulsion copolymerizations using near-infrared spectroscopy ", J. Appl. Polym. Sci., vol. 84, $n^{\circ} 14$, p. 2670- 2682, juin 2002.

[13] O. Elizalde, J. R. Leiza, et J. M. Asua, « On-line monitoring of all-acrylic emulsion polymerization reactors by Raman spectroscopy ", Macromol. Symp., vol. 206, $\mathrm{n}^{\circ}$ 1, p. 135- 148, févr. 2004. 
[14] C. Houben, G. Nurumbetov, D. Haddleton, et A. A. Lapkin, " Feasibility of the Simultaneous Determination of Monomer Concentrations and Particle Size in Emulsion Polymerization Using in Situ Raman Spectroscopy ", Ind. Eng. Chem. Res., vol. 54, n 51, p. 12867- 12876, déc. 2015.

[15] O. Elizalde, M. Azpeitia, M. M. Reis, J. M. Asua, et J. R. Leiza, " Monitoring Emulsion Polymerization Reactors: Calorimetry Versus Raman Spectroscopy ", Ind. Eng. Chem. Res., vol. 44, n 18, p. 7200- 7207, août 2005.

[16] P. D. Gossen, J. F. Macgregor, et R. H. Pelton, « Composition and Particle Diameter for Styrene/Methyl Methacrylate Copolymer Latex Using UV and NIR Spectroscopy ", Appl. Spectrosc., vol. 47, n 11, p. 1852- 1870, nov. 1993.

[17] A. Cherfi et G. Févotte, "On-line conversion monitoring of the solution polymerization of methyl methacrylate using near-infrared spectroscopy », Macromol. Chem. Phys., vol. 203, $n^{\circ}$ 9, p. 1188, juin 2002.

[18] M. M. Reis, P. H. H. Araújo, C. Sayer, et R. Giudici, "Correlation between Polymer Particle Size and in-situ NIR Spectra ", Macromol. Rapid Commun., vol. 24, n 10, p. 620-624, juill. 2003.

[19] N. Nguyen Do Trong et al., "Reprint of "Optical properties-microstructure-texture relationships of dried apple slices: Spatially resolved diffuse reflectance spectroscopy as a novel technique for analysis and process control" ", Innov. Food Sci. Emerg. Technol., vol. 24, p. 145- 153, août 2014.

[20] B. Igne, S. Talwar, H. Feng, J. K. Drennen, et C. A. Anderson, « Near-Infrared Spatially Resolved Spectroscopy for Tablet Quality Determination », J. Pharm. Sci., vol. 104, n ${ }^{\circ} 12$, p. 4074- 4081, déc. 2015.

[21] M. Rey-Bayle et al., "Potential of vis-NIR spectroscopy to monitor the silica precipitation reaction ", Anal. Bioanal. Chem., vol. 409, n³ 3, p. 785- 796, janv. 2017.

[22] H. Abdi, "Partial least squares regression and projection on latent structure regression (PLS Regression): PLS REGRESSION », Wiley Interdiscip. Rev. Comput. Stat., vol. 2, n 1, p. 97- 106, janv. 2010.

[23] B. Zimmermann et A. Kohler, " Optimizing Savitzky-Golay Parameters for Improving Spectral Resolution and Quantification in Infrared Spectroscopy ", Appl. Spectrosc., vol. 67, $\mathrm{n}^{\circ}$ 8, p. 892- 902, août 2013.

[24] M.-G. Zhang, Z.-X. Weng, Z.-M. Huang, et Z.-R. Pan, "Effects of monomer polarity on MMA/BA/NaMA emulsifier-free emulsion copolymerization ", Eur. Polym. J., vol. 34, n ${ }^{\circ}$ 9, p. 1243- 1247, sept. 1998.

[26] S. G. Roos, A. H. E. Müller, et K. Matyjaszewski, "Copolymerization of $n$-Butyl Acrylate with Methyl Methacrylate and PMMA Macromonomers: Comparison of Reactivity Ratios in Conventional and Atom Transfer Radical Copolymerization ", Macromolecules, vol. 32, $\mathrm{n}^{\circ} 25$, p. 8331- 8335, déc. 1999.

[27] E. Frauendorfer, A. Wolf, et W.-D. Hergeth, "Polymerization Online Monitoring ", Chem. Eng. Technol., vol. 33, n 11, p. 1767- 1778, nov. 2010.

[28] W. K. Silva, D. L. Chicoma, et R. Giudici, "In-situ real-time monitoring of particle size, polymer, and monomer contents in emulsion polymerization of methyl methacrylate by near infrared spectroscopy ", Polym. Eng. Sci., vol. 51, n 10, p. 2024- 2034, oct. 2011.

[29] M. Rey-Bayle, R. Bendoula, N. Caillol, et J.-M. Roger, " Multiangle near infrared spectroscopy associated with common components and specific weights analysis for in line monitoring ", J. Infrared Spectrosc., p. 096703351983006, févr. 2019. 